\title{
Body temperature change in the snapping turtle (Chelydra serpentina)'
}

\author{
ROBERT L. GOSSETTE and ARTHUR HOMBACH, Compar- \\ ative Animal Behavior Laboratory, Hofstra University, \\ Hempstead, LL.I., New York 11550
}

One prominent operation for the control of motivationalincentive variables with reptilian species is peripheral body temperature change. However, little is known about the relation between peripheral temperature change, and the rate of deep body temperature adjustment, which may be more important in establishing a motivational level. Rate of deep body temperature adaptation to a peripheral change of 10 deg $F$ was determined for 13 snapping turtles ranging in body weight from 500 to $14,000 \mathrm{~g}$. Adaptation was a negatively accelerated decreasing function of body weight.

While considerable progress is being made in the comparative analysis of learned behavior of various avian and mammalian species (Warren, 1965; Gossette, 1966) and to some extent fish (Bitterman, 1965), the reptiles have been somewhat neglected. A few species of turtles (Kirk \& Bitterman, 1963) and lizards (Alkov \& Crawford, 1966) have been subjected to learning experiments and have proved to be promising Ss. Generally, however, only fragmentary data are available, but the nature of reptile learning is already assuming a role of critical systematic importance for the comparative analysis of learning. On the one hand, variation in performance with such calibration procedures as successive discrimination reversal (SDR) has been interpreted as revealing an underlying quantitative dimensional variation in "learning ability" descending through the vertebrate classes (Gossette, 1966). An alternative view (Bitterman, 1965), however, argues for a qualitative shift in learning, occurring somewhere among the reptiles. That is, reptilian performance presumably represents a transitional stage in the phylogenetic development of SDR performances.

One factor that has discouraged the study of reptilian learning has been the difficulty of assuring constant, reliable motivational and incentive controls. While food deprivation has been attempted with turtles (Bitterman, 1964) and snakes (Crawford \& Bartlett, 1966) precise control of this variable is made difficult by the widely fluctuating metabolic rates of these organisms. The ability of reptiles, especially the larger ones, to undergo extended deprivation periods is well known. Aversive thermal stimulation has been attempted with iguanas (Alkow \& Crawford, 1966) with appreciable success. Due to the ease of control of thermal stimulation, and the necessary correlation of peripheral and deep body temperatures, this variable would appear to be the most precise, efficient, and widely applicable mode of specifying motivational-incentive levels for this class of organisms. Since deviation of deep body temperature from some optimal range would appear to be the basis for thermal motivation, a preliminary step in the application of this variable in the study of reptilian learning should be the determination of how deep body temperature varies with peripheral (environmental) temperature. Since the adaptation function, that is, the rate of heat exchange between the reptilian body and its environment, is importantly determined by the S's mass, body weight should be an important factor controlling the adaptation function. Accordingly, the problem for the present experiment was the measurement of deep body temperature change for specimens of snapping turtles of various sizes.

\section{METHOD}

The Ss were 13 snapping turtles (Chelydra serpentina) varying in body weight from $500 \mathrm{~g}$ to $14,000 \mathrm{~g}$. Some of the Ss were collected locally, while others were obtained from North Carolina. All Ss were maintained for at least three months in the laboratory before testing, were eating readily, and appeared in good health at the beginning of the experiment. Two Ss, however, died from undetermined causes before the final tests were finished.

All body temperature measures were obtained through a liquid immersion thermistor cloacal probe, 0.16 in. in diam, connected to a Rustrak recorder, Model 33. Temperature values were read directly from the resulting tapes at 3-min intervals. Ss were initially maintained in a temperature stabilization tank at 74 deg $F$ until body temperature matched that of the water of the tank. At that point $S$ was quickly transferred to the test tank containing water of $64 \mathrm{deg} F$. Measurement of body temperature continued until S's temperature matched that of the water in the test tank. Body temperature measurement was replicated four times for eight SS, three times for one S, and two times for three Ss. The water level in both tanks was high enough to completely cover S's body. Motor activity for all Ss was generally slight and only occasionally produced artifactual body temperature increments.

\section{RESULTS AND DISCUSSION}

The principal measure to be reported is the rate of change of body temperature. This measure was obtained for each $\mathrm{S}$ by dividing the range of temperature reduction, e.g., $10 \mathrm{deg} F$, by the mean time required for that $S$ to stabilize its body temperature to the temperature level of the test tank water, e.g., 64 deg F. Fig. 1 shows the plot of body temperature adaptation rates as a function of body size. A best fit curve was drawn by inspection through the actual data points yielding a negatively accelerated decreasing function. While there is an insufficient number of large Ss, e.g., above $6000 \mathrm{~g}$,

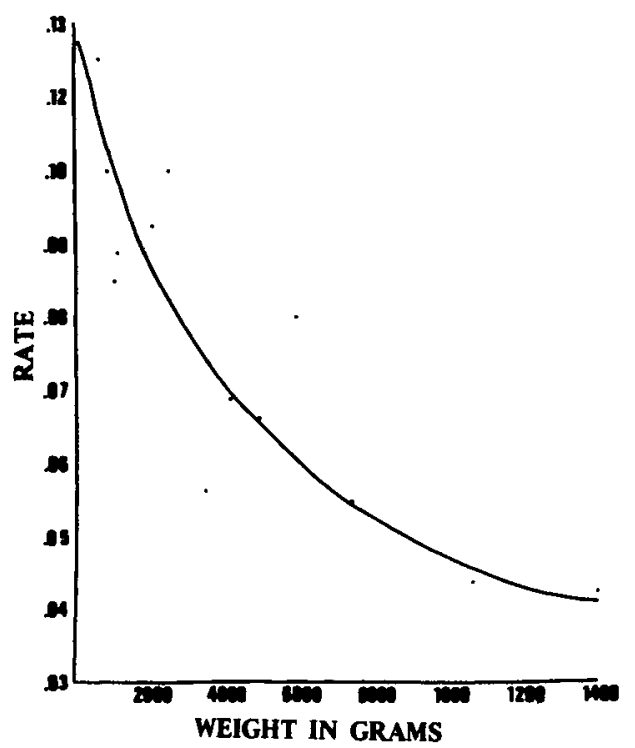

Fig. 1. Rate of body temperature change over an adaptation span of $10 \mathrm{deg} F$ as a function of body weight. 


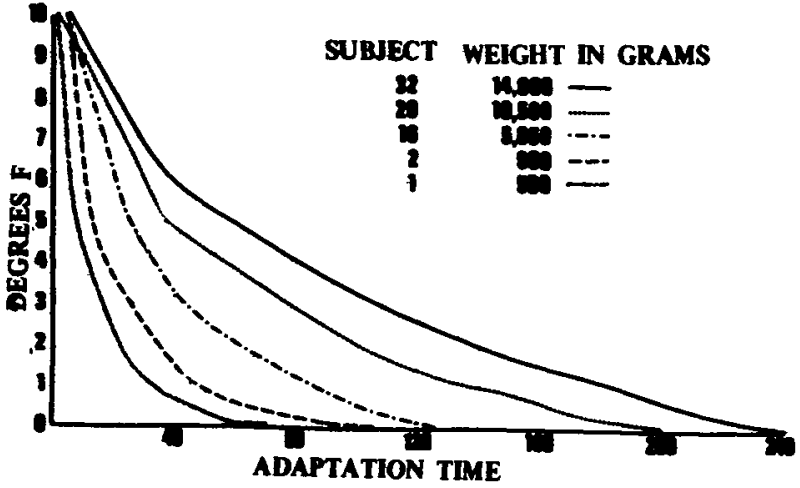

Fin. 2. Change in deep body temperature, from $74 \mathrm{deg} F$ to $64 \mathrm{deg} F$, as a function of time for selected Sa.

it would appear that differences in adaptation rates may become negligible among the largest members of the species. Fig. 2 presents individual functions for selected Ss showing the actual decline of body temperature as a function of time in the test tank. As can be seen, the time required for S's body temperature to match that of the test tank water varied appreciably, from $80 \mathrm{~min}$ for the smallest S, No. 1, to $240 \mathrm{~min}$ for the largest $\$$, No. 32.

It is readily apparent that the body size of a turtle is an important determinant of the rate of deep body temperature adaptation, particularly for smaller specimens. In addition, the time that a turtle is exposed to a medium of a given temperature prior to introduction into a new medium of a different temperature will control the time required for it to match that of the new medium. Thus, in investigations of learning of turtles, in which motivation-incentive variables are operationally defined in terms of S's peripheral temperature change, it would appear important to equate Ss for both size and exposure times.

The nature of the adaptation function suggests a potentially valuable approach to specifying and controlling both motivation and incentive levels. Ss may be maintained in a temperature controlled tank to lower their body temperature to a specified reduction level. The exposure time in the control tank, and/or the difference between optimal or maintenance temperature and control tank temperature could be the basis for assigning different motivational levels. In addition, the length of time $S$ is permitted to remain in the goal box tank, with attendant rise in body temperature, could provide a basis for specifying incentive level. At present, research is in progress to determine the relation between behavioral indices of motivation and level of body temperature reduction.

\section{REFERENCES}

ALKOV, R. A., CRA WFORD, F. T. Successive reversal training in the lizard, Iguana iguana. Proceedings of the 74th Annual Convention of the American Psychological Association 1966, 153-154.

BITTERMAN, M. E. Phyletic differences in learning. American Psy chologist, 1965, 20, 396-410.

BITTERMAN, M. E. The evolution of intelligence. Scientific American, $1965,212,92-100$.

BITTERMAN, M. E. An instrumental technique for the turtle. Journal of the Experimental Analysis of Behavior, 1964, 7, 189-190.

CRAWFORD, F. T. \& BARTLETT, C. W. Runway behavior of the gray rat snake with food and water reinforcement. Psychonomic Science, $1966,4,99-100$.

GOSSETTE, R. Comparison of successive discrimination reversal performances across 14 different avian and mammalian species. Paper given at AAAS, Division of Animal Behavior, Washington, D. C., 1966.

KIRK, K. L., BITTERMAN, M. E. Habit reversal in the turtle. Quarterly Journal of Experimental Psychology, 1963, 15, 52-57.

WARREN, J. M. Comparative psychology of learning. Annual Review of Psychology, Vol. 16. Palo Alto: Annual Reviews, Inc., 1965.

$$
\text { NOTE }
$$

1. Supported in part by NSF Grant GB 4799 awarded to the senior author. 